\title{
Air Stacking: A Detailed Look Into Physiological Acute Effects on Cough Peak Flow and Chest Wall Volumes of Healthy Subjects
}

\author{
Antonio Sarmento MSc, Armèle F Dornelas de Andrade $\mathrm{PhD}$, fllia Nadinne DF Lima PhD, \\ Andrea Aliverti PhD, Guilherme Augusto de Freitas Fregonezi PhD, and Vanessa R Resqueti PhD
}

\begin{abstract}
BACKGROUND: Air stacking (AS) is a lung insufflation method that requires the use of a manual insufflator to provide air volumes higher than inspiratory capacity. Neuromuscular patients benefit the most from the maneuver; however, the acute effects of AS in healthy subjects are still unclear. METHODS: Twenty healthy subjects ( 8 males) were studied by optoelectronic plethysmography to investigate the immediate effects of $\mathrm{AS}$ on cough peak flow, operational volume variations, distribution of these volumes in the chest wall compartments (pulmonary rib cage, abdominal rib cage, and abdominal), breathing pattern, and shortening velocity of the respiratory muscles during a protocol that included vital capacity maneuvers and spontaneous coughs before and after AS. RESULTS: Statistically significant increases in cough peak flow $(P<.03)$ and inspiratory capacity $(P<.001)$ were found immediately after AS. During its application, the pulmonary rib cage compartment was the largest contributor $(P=.002)$ to chest wall volume displacement. A significant increase in chest wall tidal volume $(P<.001)$, mainly in the pulmonary rib cage $(P<.001)$, was observed. Significant increases $(P<.001)$ in end-inspiratory chest wall volume were observed with main distribution in pulmonary $(P<.001)$ and abdominal rib cage $(P=.01)$. Significant increases in shortening velocity index of inspiratory muscles $(P<.001)$, expiratory muscles $(P<.001)$, and diaphragm $(P<.001)$ were also observed. In addition, significant decreases in expiratory time $(P<.001)$ and increases in duty cycle $(P=.02)$, breathing frequency $(P=.02)$, minute ventilation $(P<.001)$, maximum inspiratory $(P<.001)$, and expiratory flow $(P<.001)$ were observed. CONCLUSIONS: In healthy subjects, cough peak flow and chest wall volumes can be increased immediately after the application of the AS maneuver. Key words: air stacking; cough peak flow; chest wall volumes; pulmonary expansion and optoelectronic plethysmography. [Respir Care 2017;62(4):432-443. (C) 2017 Daedalus Enterprises]
\end{abstract}

\section{Introduction}

In healthy subjects, about $2.3 \mathrm{~L}$ of air flow are expelled during a normal cough from 6 to $20 \mathrm{~L} / \mathrm{s} .{ }^{1}$ Cough peak flow

Mr Sarmento, Ms Lima, Dr de Freitas Fregonezi, and Dr Resqueti are affiliated with the PneumoCardioVascular Lab, Hospital Universitário Onofre Lopes, Empresa Brasileira de Serviços Hospitalares (EBSERH), Universidade Federal do Rio Grande do Norte (UFRN), Natal RN, Brazil and the Laboratório de Desempenho PneumoCardioVascular \& Músculos Respiratórios, Departamento de Fisioterapia, Universidade Federal do Rio Grande do Norte, UFRN, Natal RN, Brazil. Dr de Andrade is affiliated with the Departamento de Fisioterapia, Universidade Federal de Pernambuco, Recife PE, Brasil. Dr Aliverti is affiliated with the Dipartimento di Elettronica, Informazione e Bioingegneria Politecnico di Milano, Milan, Italy.

Mr Sarmento presented a version of this paper at the 25th Annual Congress of the European Respiratory Society, held September 26-30, 2015, in Amsterdam, Netherlands. is the most reproducible assessment of cough strength, ${ }^{2}$ being dependent upon the generation of flow and velocity in the airways, ${ }^{3}$ elastic recoil of the lung, and chest wall forces. ${ }^{4}$ According to Smith et al, ${ }^{5}$ the higher the opera-

\footnotetext{
Dr de Freitas Fregonezi was supported by CNPq Grant 307353/2015-0, and Dr Regiane Resqueti was supported by CNPq Grant 310091/2015-2 (Conselho Nacional de Desenvolvimento Científico e Tecnológico). The authors have disclosed no conflicts of interest.

Correspondence: Vanessa Regiane Resqueti PhD, Laboratório de Desempenho PneumoCardioVascular e Músculos Respiratórios, Departamento de Fisioterapia, Universidade Federal do Rio Grande do Norte, Campus Universitário Lagoa Nova, Caixa Postal 1524, CEP 59072-970, Natal RN, Brazil. E-mail: vanessaresqueti@ hotmail.com.
}

DOI: $10.4187 /$ respcare.05189 
tional volumes, the greater the elastic recoil of the lungs and the lower the airway resistance. Thus, in restrictive diseases, high cough peak flows can be generated after an increase in lung volume ${ }^{6}$ by either passive ${ }^{7}$ (air stacking $[\mathrm{AS}]^{6,8,9}$ and mechanical infflation-exsufflation ${ }^{10-12}$ ) or active (breath-stacking ${ }^{13-15}$ and glossopharyngeal breathing ${ }^{16,17}$ ) methods.

Kang and $\mathrm{Bach}^{1}$ defined $\mathrm{AS}$ as a lung insufflation method that requires the use of a manual insufflator or a portable volume ventilator to provide volumes higher than the inspiratory capacity (IC). Therefore, after the completion of a deep and maximum breath and consequent closure of the glottis, air volumes can be stacked until reaching maximum insufflation capacity (MIC), defined as the maximum volume of air that can be held with the glottis closed after insufflations. ${ }^{18}$

Patients with restrictive lung diseases, such as neuromuscular diseases, benefit the most with the AS maneuver. In this population, increases in lung volume are strongly correlated with increases in cough peak flow. ${ }^{1,5}$ In addition, the greater the lung expansion, the better the optimization of lung recoil pressure, the cough, and consequently, the secretion extraction..$^{1,19}$

However, the acute effects of the AS maneuver in healthy subjects are unclear, so we hypothesized that because the maneuver allows the respiratory system to reach MIC, which is higher than total lung capacity (TLC), the cough peak flow and chest wall volumes of this population can also be increased. To test this idea, we studied healthy subjects using optoelectronic plethysmography, ${ }^{5,20}$ an accurate system that allows noninvasive evaluation of the mechanisms during and immediately after a cough peak flow (ie, flow and volume at the same time), during a protocol that included AS maneuvers and cough peak flows.

Thus, the main objective of the study was to evaluate the immediate effects of AS on cough peak flow of healthy subjects. Second, variations in operational volumes, distribution of these volumes in the chest wall compartments (pulmonary rib cage, abdominal rib cage, and abdominal), breathing pattern, and shortening velocity of the respiratory muscles immediately after the application of AS were also evaluated.

\section{Methods}

\section{Subjects}

The research consisted of a cross-sectional study with healthy individuals from both sexes and was carried out in the Laboratory of PneumoCardioVascular Physiotherapy and Respiratory Muscles of the Federal University of Rio Grande do Norte within the confines of the World Medical Association Declaration of Helsinki for medical research using human participants and approved by the Research

\section{QUICK LOOK}

\section{Current knowledge}

The use of the air-stacking maneuver has been studied to increase cough peak flow in a variety of neuromuscular disease patients, but not healthy subjects. In addition, differently from cough peak flow instruments, optoelectronic plethysmography allows a real-time assessment of a natural cough and chest wall volumes during and after the application of a technique without the use of mouthpieces or any interface that may alter mouth and cheeks movements.

\section{What this paper contributes to our knowledge}

The immediate application of volume by the air-stacking maneuver is able to increase chest wall volumes and cough peak flow of healthy subjects positioned at $45^{\circ}$ body inclination. The study also reveals that in this population the increase in chest wall volume (mean of $599 \mathrm{~mL}$ ) occurred above total lung capacity and resulted in no chest wall hyperinflation.

Ethics Committee (approval 1.344.512/2015). All individuals involved in the study signed a clear and informed consent form.

Subjects who self-reported as healthy with no history of smoking, heart, or lung disease were included in the study. Those who had spirometric values below predicted values $\left(<80 \%\right.$ of $\mathrm{FVC}$ and $\left.\mathrm{FEV}_{1}\right)$, did not adapt, or failed to perform the AS technique were excluded.

\section{Lung Function}

A Koko DigiDoser spirometer (nSpire Health, Longmont, Colorado) was used to perform pulmonary function test. Three technically acceptable and reproducible forced expiratory curves were obtained for each participant. Variability between them was $<5 \%$, and only the curve with the best performance was considered for analysis. $\mathrm{FVC}, \mathrm{FEV}_{1}$, and $\mathrm{FEV}_{1} / \mathrm{FVC}$ in their absolute and relative values were considered for analysis.

Technical procedures, acceptability criteria, reproducibility, and standardization of the different pieces of equipment followed the recommendations of the American Thoracic Society/European Respiratory Society. ${ }^{21}$ The predicted reference values for the studied population were calculated according to Pereira et al. ${ }^{22}$

\section{Respiratory Muscle Strength}

Respiratory muscle maximal pressures (maximum inspiratory and expiratory pressures) were performed through 


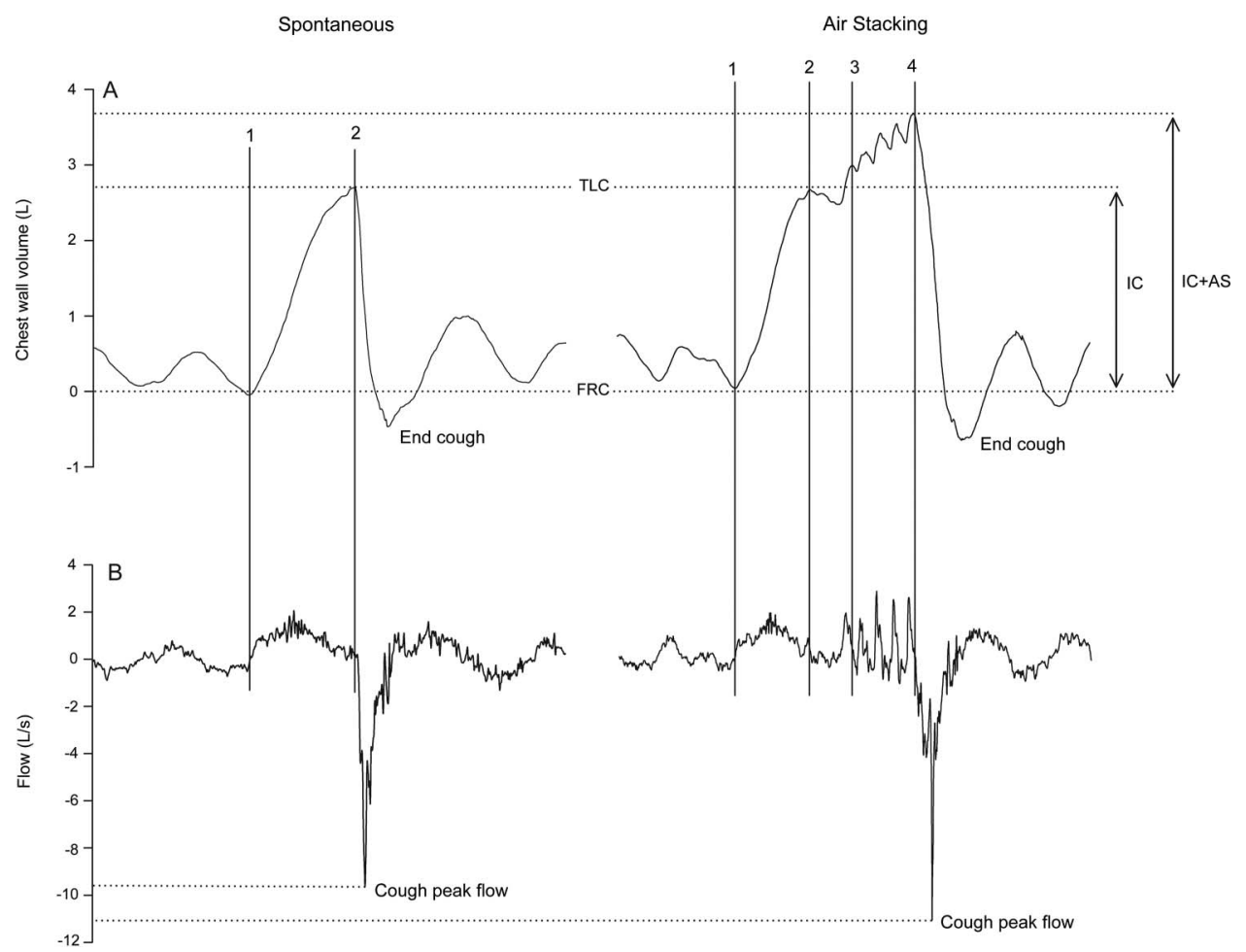

Fig. 1. Experimental tracings obtained on a representative subject during a spontaneous inspiratory capacity (IC) (1 and 2$)$ and spontaneous cough starting from TLC (left) and during application of air stacking ( 3 and 4) and subsequently cough starting from IC + AS (4) (right). On the right, IC and IC + AS volume are indicated. A: Chest wall volume variations measured by optoelectronic plethysmography. B: Flow variation measured by optoelectronic plethysmography; TLC = total lung capacity; AS = air stacking; FRC = functional residual capacity.

a digital manometer (NEPEB-LabCare, Belo Horizonte, Brazil) with the subject in a seated position and with his or her hands in a neutral position.

Maximum inspiratory pressure was obtained starting from residual volume, and maximum expiratory pressure was obtained starting from TLC. The evaluation was considered complete when 3 acceptable maneuvers were performed, of which 2 were reproducible (with variation $\leq 10 \%$ of the highest value) with $1 \mathrm{~min}$ of rest between the tests. Interpretation of results followed the recommendations proposed by Neder et $\mathrm{al}^{23}$ for the Brazilian population.

The sniff nasal inspiratory pressure test was conducted with one nostril occluded by a nose clip and performed from functional residual capacity with the subjects seated upright, their backs against a chair, knees and hips flexed to $90^{\circ}$, and their heads in a neutral position. The test was considered complete when 5 acceptable maneuvers had been performed with a 30 -s interval between them. ${ }^{24}$ Previously described reference equations ${ }^{25}$ were used for interpreting the results.

\section{Air Stacking}

A manual resuscitator (RWR, São Paulo, Brazil) was used to perform the AS technique. The sum of the inspired volume (IC) and the volume applied by AS, reaching the MIC level ${ }^{18}$, was represented as IC + AS. IC + AS was achieved with the subject taking a deep breath, starting from functional residual capacity and ending at TLC (Fig. 1, markers 1 and 2, respectively), holding it and air stacking consecutive delivered volumes of air (Fig. 1, markers 3 and 4) until the maximum volume could be held with the glottis closed. ${ }^{1}$ Each volume delivered by the manual insufflator was followed by a deep inspiration and subsequent closure of the glottis. During this moment, the subjects were instructed to not exhale, holding the amount of air in their lungs. At the end of the set of maneuvers, the mask was removed, and the subject was asked to perform a strong cough starting from IC + AS volume. The highest cough peak flow of 3 attempts was taken into account for analysis and compared with preand post-spontaneous coughs.

\section{Study Design}

For each subject, all measurements were made in a single day. After collection of anthropometric data (weight, height, and body mass index), lung function, and respiratory muscle strength, the subjects were positioned on a standard bed at $45^{\circ}$ trunk inclination, and the data were 

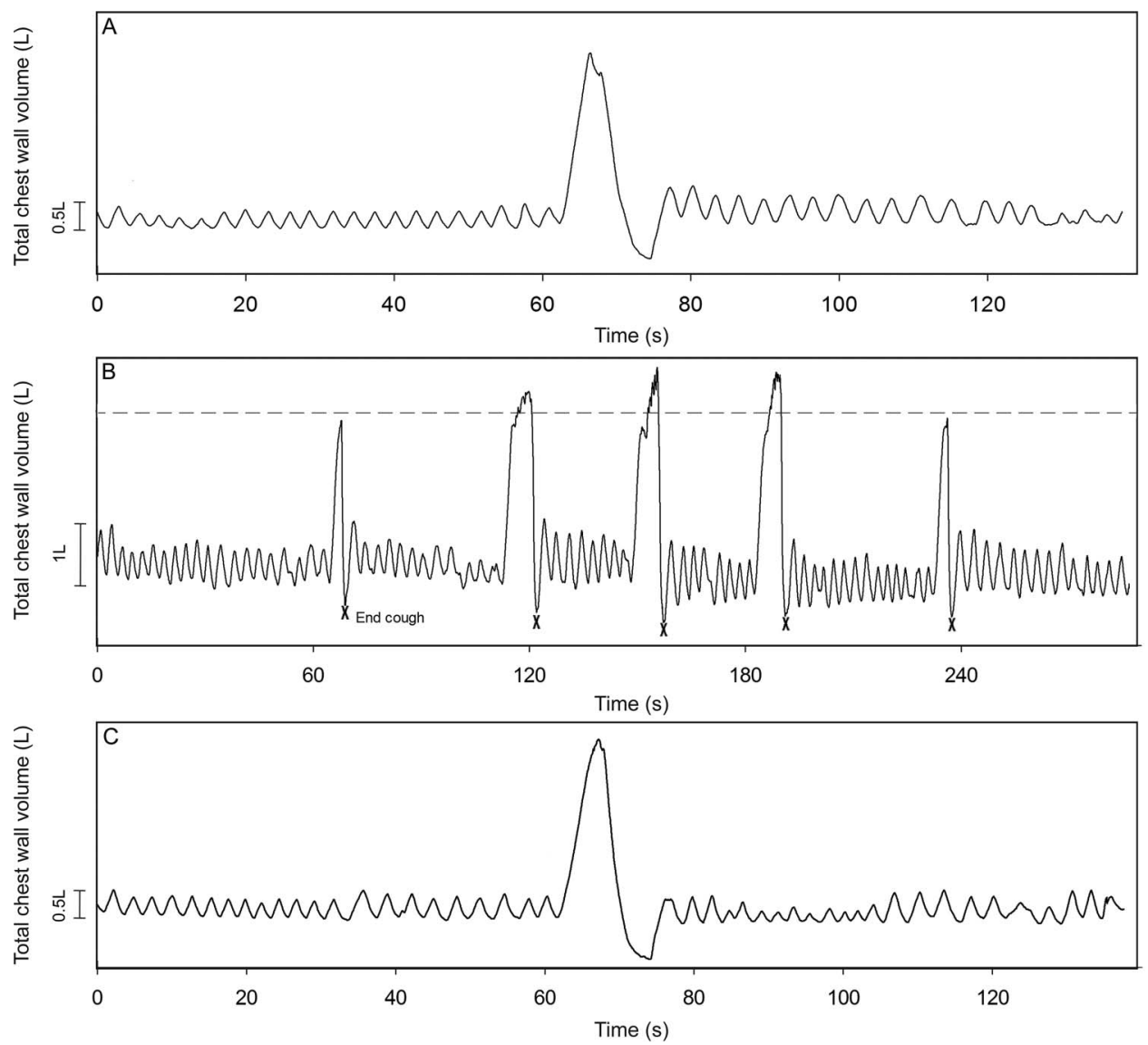

Fig. 2. Experimental tracings of total chest wall volume variation during a test on a single representative subject. The study protocol included a period of quiet breathing $(A)$ and a slow vital capacity maneuver $(C)$ and a period of quiet spontaneous breathing, spontaneous cough, and 3 sets of air stacking maneuvers interspersed with periods of quiet breathing (B). The dotted line in B denotes total lung capacity. X, end of cough.

recorded in 3 phases (Fig. 2): phase 1, (1) $60 \mathrm{~s}$ of quiet breathing (QB), (2) slow vital capacity maneuver, and (3) $60 \mathrm{~s}$ of QB; phase 2, (1) $60 \mathrm{~s}$ of QB, (2) spontaneous cough (starting from spontaneous IC), (3) $40 \mathrm{~s}$ of QB, (4) cough immediately after AS (starting from the volume reached after the insufflations [IC + AS]) (this step was repeated 3 times with an interval of $20 \mathrm{~s}$ in between), (5) $40 \mathrm{~s}$ of QB, (6) spontaneous cough (starting from spontaneous IC), and (7) $60 \mathrm{~s}$ of QB; phase 3, (1) $60 \mathrm{~s}$ of $\mathrm{QB},(2)$ slow vital capacity maneuver, and (3) $60 \mathrm{~s}$ of $\mathrm{QB}$.

\section{Assessment of Cough Peak Flow and Chest Wall Volumes}

The optoelectronic plethysmography equipment (BTS Bioengineering, Garbagnate Milanese, Italy) allowed measurement of chest-wall volume $\left(\mathrm{V}_{\mathrm{CW}}\right)$ and volume of its compartments. The equipment was calibrated before each data collection to recognize the markers and using a frequency of 60 frames/s. With the subject at $45^{\circ}$ body inclination, 52 retro-reflective markers were placed at spe- cific points of the thorax and abdomen, and 6 photosensitive cameras were positioned around the subject ( 3 on the left and 3 on the right) to capture the movement changes of the markers according to the protocol described by Aliverti et al. ${ }^{26}$ Volumes were obtained following an experimental model according to the Gauss theorem. ${ }^{20,27}$

From optoelectronic plethysmography data, the following variables were considered for further analysis: cough peak flow (derived by the volume displaced by chest wall and time during the cough $\left.\left[\Delta \mathrm{V}_{\mathrm{CW}} / \Delta \mathrm{t}\right]\right),{ }^{28,29}$ chest wall tidal volume and its compartments, IC, vital capacity, duty cycle $\left(\mathrm{T}_{\mathrm{I}} / \mathrm{T}_{\text {tot }}\right)$, maximum inspiratory $\left(\Delta \mathrm{V}_{\mathrm{T}(\mathrm{CW})} / \mathrm{T}_{\mathrm{I}}\right)$ and expiratory flow $\left(\Delta \mathrm{V}_{\mathrm{T}(\mathrm{CW})} / \mathrm{T}_{\mathrm{E}}\right)$, minute ventilation $\left(\dot{\mathrm{V}}_{\mathrm{E}}\right)$, breathing frequency, inspiratory time $\left(\mathrm{T}_{\mathrm{I}}\right)$, expiratory time $\left(T_{E}\right)$, total time of respiratory cycle $\left(T_{t o t}\right)$, end-inspiratory volume, end-expiratory volume, and percentage of contribution of the compartments to $\mathrm{V}_{\mathrm{CW}}$. Abdominal $\Delta \mathrm{V}\left(\Delta \mathrm{V}_{\mathrm{ab}}\right) / \mathrm{T}_{\mathrm{I}}$, pulmonary rib cage $\Delta \mathrm{V}\left(\Delta \mathrm{V}_{\mathrm{rcp}}\right) / \mathrm{T}_{\mathrm{I}}$, and $\Delta \mathrm{V}_{\mathrm{ab}} / \mathrm{T}_{\mathrm{E}}$ were calculated as shortening velocity index of diaphragm, inspiratory, and expiratory muscles, respectively, as described previously. ${ }^{30}$ 
Table 1. Characteristics of Each Subject in Relation to Anthropometric Data

\begin{tabular}{|c|c|c|c|c|c|}
\hline Subject & Sex & Age (y) & Height (m) & Weight $(\mathrm{kg})$ & BMI $\left(\mathrm{kg} / \mathrm{m}^{2}\right)$ \\
\hline 1 & Female & 24 & 1.76 & 70 & 22.60 \\
\hline 2 & Female & 22 & 1.53 & 50 & 21.36 \\
\hline 3 & Female & 22 & 1.72 & 80 & 27.04 \\
\hline 4 & Female & 30 & 1.65 & 58 & 21.30 \\
\hline 5 & Female & 30 & 1.59 & 55 & 21.76 \\
\hline 6 & Female & 25 & 1.69 & 69 & 24.33 \\
\hline 7 & Female & 20 & 1.64 & 54 & 20.08 \\
\hline 8 & Female & 20 & 1.64 & 60 & 22.31 \\
\hline 9 & Female & 30 & 1.65 & 55 & 20.28 \\
\hline 10 & Female & 20 & 1.55 & 53 & 22.06 \\
\hline 11 & Female & 24 & 1.54 & 61 & 25.72 \\
\hline 12 & Female & 26 & 1.77 & 64 & 20.43 \\
\hline 13 & Male & 28 & 1.78 & 78 & 24.62 \\
\hline 14 & Male & 26 & 1.82 & 76 & 22.94 \\
\hline 15 & Male & 19 & 1.70 & 69 & 23.88 \\
\hline 16 & Male & 22 & 1.86 & 81 & 23.41 \\
\hline 17 & Male & 25 & 1.80 & 77 & 23.77 \\
\hline 18 & Male & 22 & 1.69 & 63 & 22.23 \\
\hline 19 & Male & 24 & 1.82 & 86 & 25.96 \\
\hline 20 & Male & 27 & 1.73 & 90 & 30.07 \\
\hline Mean \pm SD & & $24.3 \pm 3.5$ & $1.70 \pm 0.10$ & $67.5 \pm 11.9$ & $23.31 \pm 2.50$ \\
\hline
\end{tabular}

\section{Statistical Analysis}

Normality and data distribution were verified using the Shapiro-Wilk test. For descriptive analysis, mean and SD were used when distribution was normal, and median and interquartile interval were used for non-normal distribution.

The Wilcoxon test was used for intragroup comparison related to vital capacity. Repeated measures one-way analysis of variance or the Friedman test was used to verify difference between the data of the following moments: spontaneous coughs (pre- and post-spontaneous cough) and cough with AS; spontaneous inspiratory capacities $\left(\mathrm{IC}_{\text {pre }}\right.$ and $\left.\mathrm{IC}_{\text {post }}\right)$ and $\mathrm{IC}+\mathrm{AS}$; and quiet breathing before and after cough (After ${ }_{\text {cough } 1}$, After cough2 $_{2}$, and $\mathrm{After}_{\mathrm{cough} 3}$ ) and recovery. When a significant difference was found, the Bonferroni or Dunn post hoc test was applied to identify the differences between the moments. The inferential data analysis was performed using GraphPad Prism 6.01 for Windows (GraphPad Software, La Jolla, California).

Effect sizes for the magnitude of statistically significant moment differences were calculated using Cohen's f statistic, and effect size was expressed as small $(>0.10)$, moderate (between 0.25 and 0.40 ), and large $(>0.40)$ for parametric data. Post hoc statistical power was calculated using G*Power 3.1.9.2 (Christian-Albrechts-Universität, Kiel, Germany) according to the volumes of $\mathrm{IC}_{\mathrm{pre}}$, IC $+\mathrm{AS}$, and $\mathrm{IC}_{\text {post }} . P<.05$ (2-sided) was considered as statistically significant for all statistical analysis.

\section{Results}

\section{Sample Characteristics and Power of the Study}

Twenty-one subjects were evaluated, but only 20 participated in the study ( 8 males and 12 females; mean age $24.3 \pm 3.48 \mathrm{y}$, weighing $67.51 \pm 11.91 \mathrm{~kg}$ and $1.70 \pm 0.10 \mathrm{~m}$ tall, with body mass index of $23.31 \pm 2.50$ $\mathrm{kg} / \mathrm{m}^{2}, \mathrm{FVC}$ of $4.41 \pm 0.94 \mathrm{~L}$, and $\mathrm{FEV}_{1}$ of $3.69 \pm 0.71 \mathrm{~L}$ ). One subject was excluded for presenting spirometric values below predicted. The anthropometric characteristics of the sample are shown in Table 1. Absolute and percentage of predicted values for lung function and respiratory muscle strength are shown in Table 2.

A post hoc analysis considering the calculated effect size for volumes of $\mathrm{IC}_{\mathrm{pre}}, \mathrm{IC}+\mathrm{AS}$, and $\mathrm{IC}_{\text {post }}$ moments showed a statistical power $(1-\beta)=.99$ for this study.

\section{Effects of Air Stacking on Cough Peak Flow}

During the AS maneuver (AS moment), a significant increase $(P=.03$, Cohen's $\mathrm{f}=.47$, large $)$ in cough peak flow was observed, as shown in Figure 3. Bonferroni's post hoc test showed a significant difference in AS mo- 


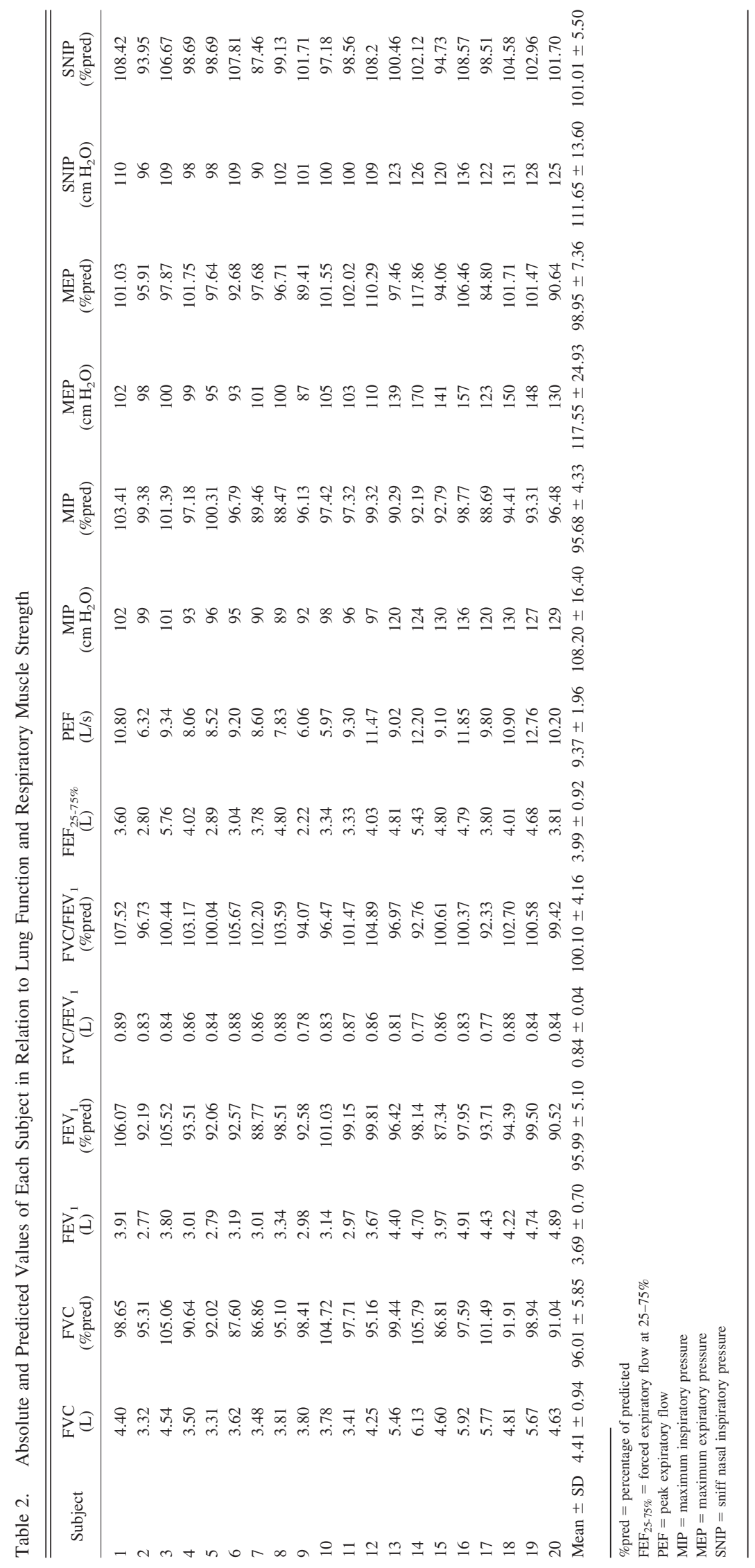



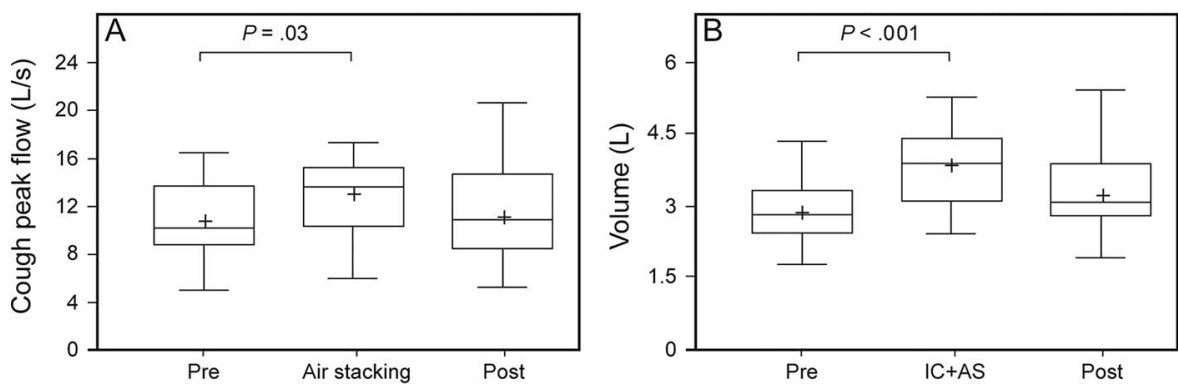

Fig. 3. Box plots showing the effects of air stacking (AS) on cough peak flow (A) and inspiratory capacity (B) of healthy subjects in 3 different moments. A: Pre = spontaneous cough before AS; Air stacking = cough immediately after AS; Post = spontaneous cough after AS. B: Pre = spontaneous inspiratory capacity before AS; IC + AS = inspiratory capacity plus AS volume; Post $=$ spontaneous inspiratory capacity after AS. Center lines indicate the median, and plus signs show mean values. The upper and lower limits of each box represent the 75th and 25th percentiles, respectively. Whiskers denote minimum and maximum values.
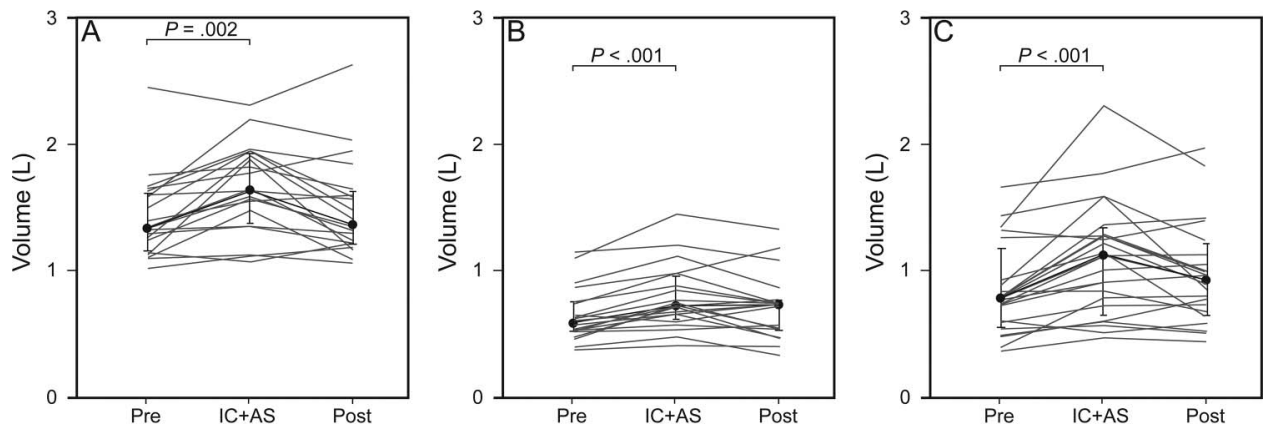

Fig. 4. Inspired volume of chest wall compartments (pulmonary rib cage [A], abdominal rib cage [B], and abdominal [C]) of all study subjects during spontaneous inspiratory capacity before (Pre), during inspiratory capacity + air stacking (IC + AS), and after (Post) the air-stacking maneuver. Black lines show median values of each compartment and interquartile ranges between 25 and $75 \%$.

ment when compared with the pre-spontaneous cough moment (mean difference of $1.76 \pm 0.08 \mathrm{~L} / \mathrm{s}$ ).

\section{Effects of Air Stacking on Inspiratory Capacity and Vital Capacity}

A significant increase in volume during the application of the maneuver $(P<.001$, Cohen's $\mathrm{f}=.82$, large $)$ was observed. Bonferroni's post hoc test showed a significant difference (mean difference of $599 \mathrm{~mL}$ ) in IC + AS compared with the $\mathrm{IC}_{\text {pre }}$ moment, with no differences between $\mathrm{IC}_{\text {post }}$ and $\mathrm{IC}_{\text {pre }}$ moments (Fig. 3).

Regarding the volume displaced by the compartments, the pulmonary rib cage compartment displaced a significantly $(P=.002)$ high amount of volume, which contributed to $40.6 \%$ of chest wall displacement during the application of the maneuver. Dunn's post hoc test showed a difference in IC + AS compared with the $\mathrm{IC}_{\text {pre }}$ moment. Significant increases in abdominal rib cage $(P<.001)$ and abdominal compartment $(P<.001$, Cohen's $\mathrm{f}=.81$, large $)$ were also observed during AS, which contributed to 23.2 and $36.2 \%$ of the total chest wall volume displacement, respectively. Dunn's and Bonferroni's post hoc test, respectively, showed a significant difference in IC + AS when compared with the $\mathrm{IC}_{\text {pre }}$ moment in both compartments (Fig. 4). No differences between IC $+\mathrm{AS}$ and $\mathrm{IC}_{\text {post }}$ moments and $\mathrm{IC}_{\text {pre }}$ and $\mathrm{IC}_{\text {post }}$ moments were found. No significant differences in vital capacity $(P=.86)$ after the application of the technique were found.

\section{Effects of Air Stacking on Total and Operational Chest Wall Volumes}

A significant increase in chest wall tidal volume $(P<$ .001) was observed after application of the maneuver. Dunn's post hoc test showed a significant increase in After cough1 $_{1}$, After cough2 $_{2}$, and After cough3 $_{3}$ moments compared with QB and recovery with a greater difference in After $_{\text {cough2 }}$ compared with the QB moment. Regarding the volume of the compartments involved, a greater increase in tidal volume of pulmonary rib cage compartment $(P<$ .001 , Cohen's $\mathrm{f}=.89$, large) was observed, followed by an increase in abdominal $(P<.001)$ and abdominal rib cage compartment $(P<.001)$. After Dunn's post hoc test, the increase in volumes of pulmonary and abdominal rib cage compartments occurred in After $\mathrm{cough}_{1}, \mathrm{After}_{\mathrm{cough} 2}$, and After $_{\text {cough3 }}$ moments compared with QB and in After ${ }_{\text {cough1 }}$ and After ${ }_{\text {cough2 } 2}$ compared with the recovery moment. In- 
Table 3. Effects of Air Stacking on Total Chest Wall and Compartmental Volumes and Operational Volumes

\begin{tabular}{|c|c|c|c|c|c|c|}
\hline Parameter & Quiet Breathing & After $_{\text {cough } 1}$ & After $_{\text {cough } 2}$ & After $_{\text {cough } 3}$ & Recovery & $P$ \\
\hline $\mathrm{V}_{\mathrm{T}(\mathrm{CW})}, \mathrm{L}$ & $0.40(0.32-0.50)$ & $0.61(0.50-0.79)^{* \dagger}$ & $0.57(0.43-0.91)^{* \dagger}$ & $0.55(0.40-0.71)^{* \dagger}$ & $0.41(0.32-0.59)$ & $<.001$ \\
\hline $\mathrm{V}_{\mathrm{T}(\mathrm{rcp})}, \mathrm{L}$ & $0.14(0.09-0.20)$ & $0.30(0.19-0.40)^{* \dagger}$ & $0.28(0.16-0.43)^{* \dagger}$ & $0.22(0.13-0.37)^{*}$ & $0.18(0.08-0.26)$ & $<.001$ \\
\hline $\mathrm{V}_{\mathrm{T}(\mathrm{rca})}, \mathrm{L}$ & $0.06(0.05-0.07)$ & $0.10(0.09-0.13)^{* \dagger}$ & $0.09(0.07-0.17)^{* \dagger}$ & $0.10(0.07-0.14)^{*}$ & $0.06(0.05-0.10)$ & $<.001$ \\
\hline $\mathrm{V}_{\mathrm{T}(\mathrm{ab})}, \mathrm{L}$ & $0.20(0.13-0.24)$ & $0.21(0.18-0.28) \dagger$ & $0.22(0.18-0.29) \dagger$ & $0.20(0.13-0.31)$ & $0.18(0.13-0.22)$ & $<.001$ \\
\hline $\mathrm{V}_{\mathrm{T}(\mathrm{rcp})}, \% \doteqdot$ & $35.82(27.05-47-94)$ & $49.81(29.20-56.57)^{* \dagger}$ & $48.73(32.55-57.26)^{*}$ & $43.87(2886-54.67)^{*}$ & $43.57(26.05-53.83)$ & $<.001$ \\
\hline $\mathrm{V}_{\mathrm{T}(\mathrm{rca})}, \% \neq$ & $16.61(14.93-17.97)$ & $17.90(15.86-19.07)$ & $17.71(15.65-19.34)$ & $17.56(15.80-18.90)$ & $17.32(15.05-19.13)$ & .061 \\
\hline $\mathrm{V}_{\mathrm{T}(\mathrm{ab})}, \% \ddagger$ & $47.65(37.14-55.71)$ & $33.19(22.88-51.36)^{*}$ & $34.60(25.77-49.85)^{*}$ & $40.68(27.73-54.15)^{*}$ & $39.24(29.90-56.49)$ & $<.001$ \\
\hline $\mathrm{EIV}_{\mathrm{CW}}, \mathrm{L}$ & $11.30(10.38-12.64)$ & $11.57(10.61-13.17)^{*}$ & $11.87(10.95-13.08)^{*}$ & $11.81(10.82-13.07)^{*}$ & $11.45(10.47-13.70)$ & $<.001$ \\
\hline $\mathrm{EIV}_{\mathrm{rcp}}, \mathrm{L} \ddagger$ & $5.69(5.15-6.29)$ & $5.83(5.33-6.39)^{*}$ & $5.76(5.38-6.37)^{*}$ & $5.72(5.27-6.33)^{*}$ & $5.73(5.20-6.34)^{*}$ & $<.001$ \\
\hline $\mathrm{EIV}_{\text {rca }}, \mathrm{L}$ & $1.94(1.64-2.28)$ & $2.02(1.72-2.34)$ & $2.08(1.75-2.33)^{*}$ & $2.04(1.76-2.33)$ & $2.00(1.69-2.31)$ & .01 \\
\hline $\mathrm{EIV}_{\mathrm{ab}}, \mathrm{L}$ & $3.86(3.41-4.58)$ & $3.82(3.41-4.61)$ & $3.82(3.47-4.57)$ & $3.80(3.49-4.67)$ & $3.80(3.41-4.56)$ & .56 \\
\hline $\mathrm{EEV}_{\mathrm{CW}}, \mathrm{L}$ & $10.92(10.08-12.08)$ & $10.93(10.09-12.20)$ & $10.92(10.07-12.16)$ & $10.99(10.13-12.15)$ & $11.05(10.10-12.17)$ & .10 \\
\hline $\mathrm{EEV}_{\mathrm{rcp}}, \mathrm{L} \ddagger$ & $5.02(5.52-6.14)$ & $5.47(5.02-6.15)$ & $5.49(4.98-6.13)$ & $5.53(5.01-6.12)$ & $5.55(5.04-6.12)$ & .15 \\
\hline $\mathrm{EEV}_{\text {rca }}, \mathrm{L}$ & $1.86(1.58-2.19)$ & $1.91(1.61-2.22)$ & $1.91(1.64-2.20)$ & $1.92(1.63-2.19)$ & $1.91(1.64-2.21)$ & .39 \\
\hline $\mathrm{EEV}_{\mathrm{ab}}, \mathrm{L}$ & $3.67(3.27-4.31)$ & $3.67(3.25-4.32)$ & $3.64(3.24-4.32)$ & $3.66(3.25-4.32)$ & $3.64(3.26-4.34)$ & .17 \\
\hline
\end{tabular}

Data are presented as median (interquartile range)

* Intragroup difference compared with quiet breathing.

$\dagger$ Intragroup difference compared with recovery.

$\$$ Parametric data distribution.

$\mathrm{V}_{\mathrm{T}}=$ tidal volume

$\mathrm{CW}=$ chest wall

$\mathrm{rcp}=$ pulmonary rib cage

rca $=$ abdominal rib cage

$\mathrm{ab}=$ abdominal

EIV $=$ end-inspiratory volume

$\mathrm{EEV}=$ end-expiratory volume

creases in tidal volume of the abdominal compartment were observed in After $r_{\text {cough } 1}$ and After cough2 $_{2}$ compared with the recovery moment (Table 3 ).

Regarding operational volumes, a significant increase in end-inspiratory chest wall volume $(P<.001)$ was found. After Dunn's post hoc test, it was observed that the increase occurred in After ${ }_{\text {cough } 1}$, After ${ }_{\text {cough2 }}$, and After cough3 $_{\text {ch }}$ moments compared with QB. Concerning compartmental analysis, an increase in pulmonary rib cage $(P<.001$, Cohen's $\mathrm{f}=.88$, large $)$ and abdominal rib cage $(P=.01)$ was observed with no significant changes in the abdominal compartment $(P=.56)$. Regarding pulmonary rib cage, Bonferroni post hoc analysis showed a significant difference in $\mathrm{After}_{\text {cough } 1}, \mathrm{After}_{\mathrm{cough} 2}, \mathrm{After}_{\mathrm{cough} 3}$, and recovery moments compared with QB. Dunn's post hoc analysis showed a significant difference in After ${ }_{\text {cough2 } 2}$ compared with QB moment for the abdominal rib cage compartment. No significant differences were found in end-expiratory chest wall volume $(P=.10)$ or compartmental analysis (Table 3).

\section{Effects of Air Stacking on the Contribution of the Compartments to Tidal Volume}

A significant increase in the percentage of contribution of the pulmonary rib cage $(P<.001$, Cohen's $\mathrm{f}=.60$, large) to tidal volume was observed. The Bonferroni post hoc test showed a significant difference in $A_{\text {fter }}$ cough1, $\mathrm{After}_{\text {cough2 }}$, and $\mathrm{After}_{\text {cough3 }}$ compared with QB with the greatest difference in After ${ }_{\text {cough1 }}$ compared with QB moment and After cough $1_{1}$ compared with the recovery moment. No significant differences were observed related to abdominal rib cage compartment $(P=.061$, Cohen's $\mathrm{f}=.37$, moderate). Contrastingly, there was a decrease in the percentage of contribution of the abdominal compartment to tidal volume $(P<.001$, Cohen's $\mathrm{f}=.62$, large $)$. The Bonferroni post hoc test showed a significant difference in After $_{\text {cough } 1}$, After cough2 $_{2}$, and After cough3 $_{3}$ compared with QB, with the greatest difference in After cough1 $_{\text {compared with }}$ QB moment (Table 3).

\section{Effects of Air Stacking on Breathing Pattern}

A significant decrease in $\mathrm{T}_{\mathrm{E}}(P<.001)$ was observed after the application of the maneuver. Dunn's post hoc test showed that this decrease occurred in the recovery moment compared with QB. A significant increase in $\mathrm{T}_{\mathrm{I}} / \mathrm{T}_{\text {tot }}$ $(P=.02$, Cohen's $\mathrm{f}=.42$, large $)$ was also observed in After $_{\text {cough3 } 3}$ compared with the QB moment. No significant changes were observed in relation to $\mathrm{T}_{\mathrm{I}}$ and $\mathrm{T}_{\text {tot }}$ (Table 4).

A significant increase in breathing frequency $(P=.02)$ was observed. Dunn's post hoc test showed a significant 
Table 4. Effects of Air Stacking on Breathing Pattern and Shortening Velocity Index of Respiratory Muscles

\begin{tabular}{|c|c|c|c|c|c|c|}
\hline Parameter & Quiet Breathing & After $_{\text {cough1 }}$ & After $_{\text {cough2 }}$ & After ${ }_{\text {cough } 3}$ & Recovery & $P$ \\
\hline $\mathrm{T}_{\mathrm{I}}, \mathrm{s}$ & $1.72(1.35-1.88)$ & $1.53(1.26-2.04)$ & $1.39(1.18-1.84)$ & $1.47(1.28-1.87)$ & $1.52(1.22-1.83)$ & .45 \\
\hline $\mathrm{T}_{\mathrm{E}}, \mathrm{s}$ & $2.17(1.89-2.92)$ & $1.9(1.47-2.57)$ & $1.87(1.40-2.49)$ & $1.76(1.45-2.15)^{*}$ & $1.68(1.47-2.14)^{*}$ & $<.001$ \\
\hline $\mathrm{T}_{\text {tot }}, \mathrm{s}$ & $3.82(3.22-4.66)$ & $3.35(2.98-4.60)$ & $3.24(2.60-4.58)$ & $3.21(2.71-3.75)$ & $3.24(2.73-4.09)$ & .07 \\
\hline $\begin{array}{l}\text { Breathing frequency, } \\
\text { breaths/min }\end{array}$ & $16.51(14.33-19.37)$ & $18.37(13.43-20.98)$ & $18.73(13.20-23.71)$ & $18.91(16.22-23.32)^{*}$ & $19.12(14.78-22.47)$ & .02 \\
\hline$\dot{\mathrm{V}}_{\mathrm{E}}, \mathrm{L} / \mathrm{min}$ & $6.34(5.02-7.28)$ & $10.09(7.65-14.54)^{* \dagger}$ & $10.20(7.57-15.93)^{* \dagger}$ & $10.24(8.48-12.30)^{* \dagger}$ & $8.15(6.24-9.98)$ & $<.001$ \\
\hline$\Delta \mathrm{V}_{\mathrm{T}(\mathrm{CW})} / \mathrm{T}_{\mathrm{I}}, \mathrm{L} / \mathrm{s}$ & $0.26(0.20-0.31)$ & $0.39(0.29-0.53)^{* \dagger}$ & $0.39(0.30-0.58)^{* \dagger}$ & $0.40(0.33-0.44)^{*}$ & $0.30(0.22-0.39)$ & $<.001$ \\
\hline$\Delta \mathrm{V}_{\mathrm{T}(\mathrm{CW})} / \mathrm{T}_{\mathrm{E}}, \mathrm{L} / \mathrm{s}$ & $0.18(0.15-0.22)$ & $0.31(0.25-0.45)^{* \dagger}$ & $0.32(0.22-0.52)^{* \dagger}$ & $0.33(0.27-0.39)^{* \dagger}$ & $0.26(0.20-0.30)$ & $<.001$ \\
\hline $\mathrm{T}_{\mathrm{I}} / \mathrm{T}_{\text {tot }} \ddagger$ & $42.51(39.67-44.70)$ & $45.05(42.01-46.24)$ & $42.50(40.82-46.83)$ & $45.82(43.84-47.64)^{*}$ & $46.41(43.28-49.62)$ & .02 \\
\hline$\Delta \mathrm{V}_{\mathrm{rcp}} / \mathrm{T}_{\mathrm{I}}, \mathrm{L} / \mathrm{s}$ & $0.09(0.06-0.12)$ & $0.19(0.09-0.29)^{* \dagger}$ & $0.19(0.10-0.31)^{* \dagger}$ & $0.15(0.08-0.22)^{*}$ & $0.12(0.07-0.20)$ & $<.001$ \\
\hline$\Delta \mathrm{V}_{\mathrm{ab}} / \mathrm{T}_{\mathrm{I}}, \mathrm{L} / \mathrm{s} \ddagger$ & $0.08(0.06-0.10)$ & $0.11(0.07-0.15)^{*}$ & $0.11(0.07-0.17) \dagger$ & $0.11(0.07-0.18)$ & $0.11(0.06-0.14)$ & $<.001$ \\
\hline$\Delta \mathrm{V}_{\mathrm{ab}} / \mathrm{T}_{\mathrm{E}}, \mathrm{L} / \mathrm{s}$ & $0.11(0.09-0.16)$ & $0.15(0.11-0.20)$ & $0.15(0.11-0.21)$ & $0.13(0.09-0.18)$ & $0.12(0.10-0.16)$ & $<.001$ \\
\hline $\begin{array}{l}\text { Data are presented as median } \\
\text { * Intragroup difference comp } \\
\dagger \text { Intragroup difference comp } \\
\text { † Parametric data distribution } \\
\mathrm{T}_{\mathrm{I}}=\text { inspiratory time } \\
\mathrm{T}_{\mathrm{E}}=\text { expiratory time } \\
\mathrm{T}_{\mathrm{tot}}=\text { total respiratory cycle } \\
\mathrm{V}_{\mathrm{E}}=\text { minute volume } \\
\Delta \mathrm{V}_{\mathrm{T}}\left(\mathrm{CW} / \mathrm{T}_{\mathrm{I}}=\text { maximum insp }\right. \\
\Delta \mathrm{V}_{\mathrm{T}}(\mathrm{CW}) / \mathrm{T}_{\mathrm{E}}=\text { maximum exp } \\
\mathrm{T}_{\mathrm{I}} / \mathrm{T}_{\mathrm{tot}}=\text { duty cycle } \\
\Delta \mathrm{V}_{\mathrm{rcp}} / \mathrm{T}_{\mathrm{I}}=\text { shortening velocit } \\
\Delta \mathrm{V}_{\mathrm{ab}} / \mathrm{T}_{\mathrm{I}}=\text { shortening velocit } \\
\Delta \mathrm{V}_{\mathrm{ab}} / \mathrm{T}_{\mathrm{E}}=\text { shortening velocit }\end{array}$ & $\begin{array}{l}\text { (interquartile range). } \\
\text { red with quiet breathing. } \\
\text { red with recovery. } \\
\text { ime } \\
\text { iratory flow } \\
\text { ratory flow } \\
\text { y index of inspiratory muscles } \\
\text { index of diaphragm } \\
\text { y index of expiratory muscles }\end{array}$ & & & & & \\
\hline
\end{tabular}

difference in After cough3 $_{3}$ when compared with the QB moment. Significant increases in $\dot{\mathrm{V}}_{\mathrm{E}}(P<.001)$ were also observed. Dunn's post hoc test showed a significant difference in $\mathrm{After}_{\mathrm{cough} 1}$, After $\mathrm{cough}_{2}$, and After $\mathrm{Cough}_{3}$ compared with QB and recovery moments, with a greater difference observed in After $\mathrm{A}_{\text {cough2 }}$ compared with the QB moment (Table 4).

An increase in $\Delta \mathrm{V}_{\mathrm{T}(\mathrm{CW})} / \mathrm{T}_{\mathrm{I}}(P<.001)$ and $\Delta \mathrm{V}_{\mathrm{T}(\mathrm{CW})} / \mathrm{T}_{\mathrm{E}}$ $(P<.001)$ was also observed after application of the maneuver. Dunn's post hoc test showed a significant difference in After ${ }_{\text {cough } 1}$, After cough2 $_{2}$, and After cough3 $_{\text {moments }}$ compared with the QB moment and in After ${ }_{\text {cough } 1}$ and After ${ }_{\text {cough2 }}$ compared with the recovery moment for $\Delta \mathrm{V}_{\mathrm{T}(\mathrm{CW})} / \mathrm{T}_{\mathrm{I}}$, as well as in After ${ }_{\text {cough } 1}$, After $\mathrm{cough} 2_{\text {, }}$, and After $r_{\text {cough3 }}$ moments compared with the $\mathrm{QB}$ and recovery moments for $\Delta \mathrm{V}_{\mathrm{T}(\mathrm{CW})} / \mathrm{T}_{\mathrm{E}}$ (Table 4).

\section{Effects of Air Stacking on Shortening Velocity Index of Respiratory Muscles}

A significant increase in the $\Delta \mathrm{V}_{\mathrm{ab}} / \mathrm{T}_{\mathrm{I}}(P<.001$, Cohen's $\mathrm{f}=.55$, large $), \Delta \mathrm{V}_{\mathrm{rcp}} / \mathrm{T}_{\mathrm{I}}(P<.001)$, and $\Delta \mathrm{V}_{\mathrm{ab}} / \mathrm{T}_{\mathrm{E}}$ $(P<.001)$ was found. Dunn's post hoc test showed a significant difference in $\mathrm{After}_{\text {cough } 1}$, After $\mathrm{cough}_{\text {cond }}$, andter cough3 $_{3}$ compared with the QB moment and in After cough1 $_{1}$ and After $r_{\text {cough2 }}$ compared with the recovery moments for $\Delta \mathrm{V}_{\mathrm{rcp}} / \mathrm{T}_{\mathrm{I}}$, as well as $A f t e r_{\text {cough } 1}$, After ${ }_{\text {cough2 }}$, and
After ${ }_{\text {cough3 } 3}$ compared with the QB moment for $\Delta \mathrm{V}_{\mathrm{ab}} / \mathrm{T}_{\mathrm{E}}$. Regarding $\Delta \mathrm{V}_{\mathrm{ab}} / \mathrm{T}_{\mathrm{I}}$, the Bonferroni post hoc test showed a significant difference in After $_{\text {cough1 }}$ compared with QB and After cough2 $_{2}$ compared with the recovery moment (Table 4).

\section{Discussion}

The main findings of the study were that AS in healthy subjects was able to (1) increase cough peak flow immediately after the maneuver; (2) exceed inspiratory capacity, reaching the maximum insufflation capacity (IC + AS); (3) increase chest wall tidal volume with no significant changes in end-expiratory volumes; (4) modify breathing pattern; and (5) increase the shortening velocity index of respiratory muscles.

To date, this is the first study in which measurements of chest wall volumes and percentage of contribution of the chest wall compartments to tidal volume obtained after the AS maneuver through optoelectronic plethysmography were performed. Different from previous studies about AS, ${ }^{6-9,17}$ the choice of the optoelectronic plethysmography system as an evaluation tool of the cough peak flow was due to the high and noninvasive accuracy that it provides ${ }^{20}$ with no interference of mouthpieces, nose clips, face masks, or any interface that may alter mouth and cheek movements, thus ensuring a natural cough. ${ }^{28}$ Otherwise, we 
would not have any opportunity to evaluate the real alterations in chest wall kinematics or the real immediate effects of the AS maneuver on cough peak flow.

According to the effect size and power calculated, ${ }^{31}$ the population of this study was satisfactory, and the application of the maneuver resulted in a large effect on the IC of the analyzed subjects. Sivasothy et al ${ }^{32}$ showed that cough peak flow improvements could be achieved in healthy subjects, neuromuscular disease subjects, and COPD subjects when initiated from maximum inspiratory volumes. Additionally, Smith et $\mathrm{al}^{5}$ observed in healthy subjects that the greater the inspired volume, the greater the cough peak flow, with the inspired volume being the main factor in determining the expelled volume during the cough and achieved cough peak flow.

Increases in volumes beyond IC promote increases in cough peak flow, ${ }^{1}$ and although our study was performed in healthy subjects with no previous lung or rib cage disorders, the findings coincide with those of Ishikawa et al, ${ }^{4}$ where AS promoted a significant increase in the cough peak flow of 61 subjects with Duchenne muscular dystrophy. Similarly, Jeong and $\mathrm{Yoo}^{33}$ and Kang and Bach ${ }^{1,9}$ observed a significant increase in cough peak flow of subjects with cervical spinal cord injury and neuromuscular disease, respectively, after a daily program composed of AS maneuvers, attributing this increase to the increase in IC.

The volume applied through AS was able to exceed $20.4 \%$ (mean of $599 \mathrm{~mL}$ ) of the volume of $\mathrm{IC}_{\text {pre }}$ of the subjects, and this perhaps explains the fact that the highest difference was found in cough peak flows obtained immediately after the maneuver. This increase in volume above $\mathrm{IC}_{\text {pre }}$ occurred mainly in the pulmonary rib cage compartment and contributed to $40.6 \%$ of chest wall displacement, followed by the abdominal compartment (36.2\%). Furthermore, although it was nonsignificant, $78 \%$ of the subjects remained with high volumes after a rest period $\left(\mathrm{IC}_{\text {post }}\right.$ moment), and this increase may also have influenced the increase in spontaneous cough peak flows of 55\% of the subjects (post-spontaneous cough moment). The results demonstrate that the AS maneuver can increase the cough peak flow of healthy subjects, and this condition, which occurs immediately after AS, may also continue for a certain period of time. This can be explained by the fact that the positive applied pressure increases lung volumes to their maximum, causing an increase in passive elastic recoil of the lungs and rib cage, thereby decreasing the airway resistance, $4,5,19$ in addition to lengthening the expulsive phase ${ }^{5}$ and increasing the cough.

In patients with neuromuscular disease, the increase in cough peak flow after AS occurs in those for whom vital capacity changes with the application of the positive pressure. ${ }^{1,9}$ This may not happen the same way in healthy subjects, since no differences in vital capacity were found after the maneuver. Thus, we presume that the technique does not influence the vital capacity of healthy subjects, possibly because they do not present lung or chest wall disorders, thus confirming the fact that the maneuver influences the cough peak flow of this population only when a positive pressure is applied above IC level.

An increase in chest wall tidal volume throughout all of the intervention protocol was higher among After $_{\text {cough2 }}$ compared with the QB moment. This increase was mainly observed in the pulmonary rib cage followed by the abdominal and abdominal rib cage compartments, occurring in a gradual manner during the application of the maneuver and returning to baseline values after a period of quiet breathing (recovery moment). The pulmonary rib cage compartment was responsible for the largest increase in percentage of volume contribution to the chest wall, mainly between After cough1 $_{\text {and }}$ QB moments, whereas a decrease in the same proportion in the contribution of the abdominal compartment was observed during the same moments (Table 3). The increase in volume of the upper chest region may have also occurred because of the influence of the position at $45^{\circ}$ body inclination. The complacency of these compartments and the geometry of the diaphragm change with different positions, ${ }^{34}$ so the greater the body inclination (from supine to seated position), the greater the contribution of the rib cage, and the lower the contribution of the abdomen to tidal volume. ${ }^{35}$

The pulmonary rib cage compartment was mainly responsible for the increase in end-inspiratory chest wall volume, especially among After ${ }_{\text {cough2 }}$ compared with the QB moment. The same behavior was observed in the abdominal rib cage compartment with an increase in After ${ }_{\text {cough3 } 3}$ compared with the QB moment. No significant changes in end-expiratory chest wall volume or its compartments were observed, suggesting that the application of the maneuver in healthy subjects positioned at $45^{\circ}$ body inclination influences an increase in volume of the upper chest wall and does not result in chest wall hyperinflation at any time.

Because end-expiratory chest wall volume (an index of lung volume) did not change, we can presume that the increase of $599 \mathrm{~mL}$ during the application of AS (IC + AS moment) in healthy subjects was momentarily due to an increase in TLC. This fact corroborates with other studies $^{36-38}$ in which the TLC of healthy subjects can be altered after an increase in volume, and consequently transpulmonary pressure, greater than to those which the lung would normally be exposed to. Conventionally, TLC is the point of maximum inflation, and this volume is reached when maximal inspiratory muscle tension is exerted. To increase lung volume above normal TLC, the applied pressure has to be substantially high because of the low compliance at high lung volumes. ${ }^{37}$ 
The application of the maneuver also influenced changes in breathing pattern. The increase in $\dot{V}_{\mathrm{E}}$ was possibly more due to the increase in tidal volume than increases in breathing frequency. Higher expiratory flows were expelled in a shorter time (decrease in $\mathrm{T}_{\mathrm{E}}$ ), and, although $\mathrm{T}_{\mathrm{I}}$ remained constant, gradual increases in $\mathrm{V}_{\mathrm{T}(\mathrm{CW})} / \mathrm{T}_{\mathrm{I}}$ were observed, possibly due to the increase in tidal volume. This behavior may be explained by a possible change in respiratory drive caused by the applied positive pressure. $T_{\text {tot }}$ and $T_{I}$ showed no significant changes, but their small variations may have influenced the significant increase in $T_{\mathrm{I}} / \mathrm{T}_{\text {tot }}$.

Regarding the shortening velocity index of the respiratory muscles, the increase in $\Delta \mathrm{V}_{\mathrm{ab}} / \mathrm{T}_{\mathrm{I}}$ mainly occurred from an increase in volume displacement by the abdominal compartment, since no significant decrease in $T_{I}$ was found. Since the diaphragm acts as the main flow generator, ${ }^{39}$ it is likely that the increase in diaphragm shortening velocity could also influence the generation of high cough peak flow. On the other hand, the increase in $\Delta \mathrm{V}_{\mathrm{ab}} / \mathrm{T}_{\mathrm{E}}$ mainly occurred from a decrease in $\mathrm{T}_{\mathrm{E}}$ throughout application of the protocol, in addition to a higher amount of volume displaced by the compartment. In contrast to diaphragm and expiratory muscles, the inspiratory muscles of the rib cage develop more passive tension for active force development when stretched beyond their optimal size, ${ }^{40}$ and perhaps this is why a greater shortening velocity was seen in $\Delta \mathrm{V}_{\mathrm{rcp}} / \mathrm{T}_{\mathrm{I}}$ in relation to the other indexes. In general, the increase in shortening velocity could be explained by the activation and recruitment of motor units in addition to the change in neural drive. ${ }^{30}$ The neural drive to a muscle results in a power output that is the product of the developed force and the shortening velocity. Thus, the partitioning of power between force and velocity is primarily a function of the load the muscle contracts against, although central drive can determine recruitment patterns of motor units. ${ }^{41}$

According to Kang and Bach, ${ }^{9}$ for up to $50 \mathrm{y}$, no barotraumas or any complications have been reported by neuromuscular patients as a result of AS application. Although this study has been performed in healthy subjects with no primary intrinsic lung disease, none of them reported any complications during or after the application of the maneuver, since the manual insufflator was well calibrated, and the pressure valve automatically opened when reaching $40 \mathrm{~cm} \mathrm{H}_{2} \mathrm{O}$ while applying positive pressure.

The posture in $45^{\circ}$ body inclination was adopted in this study to mimic the posture in which AS is applied in most bedridden patients, so no other postures were included in the study design. Furthermore, because we only studied healthy subjects, the applicability of our results to individuals with neuromuscular disease is unknown, since they present with lung and chest wall mechanics quite different from those of healthy subjects. In addition, as limitations of the study, we can cite the lack of hemodynamic mon- itoring during AS application, pressure-volume curve and alveolar recruitment measurement, calculation of the volume and pressure at the mouth, the amount of gas compression inside the chest wall caused by $\mathrm{AS}^{42}$ as well as blood shift from the trunk to the extremities during and after application of the maneuver.

\section{Conclusions}

In healthy subjects, the cough peak flow can be increased immediately after the application of the AS maneuver, as well as the total and operational chest wall volumes, inspiratory capacity, respiratory muscle shortening velocity, and breathing pattern. Although this study has attempted to answer some questions about the AS maneuver, we do not know to what extent the technique influences lung and chest wall compliance. For this, future studies are needed to better understand the physiology around the AS maneuver in healthy subjects and subjects with restrictive lung disorders.

\section{REFERENCES}

1. Kang SW, Bach JR. Maximum insufflation capacity. Chest 2000; 118(1):61-65.

2. Salam A, Tilluckdharry L, Amoateng-Adjepong Y, Manthous CA. Neurologic status, cough, secretions and extubation outcomes. Intensive Care Med 2004;30(7):1334-1339.

3. Freitas FS, Ibiapina CC, Alvim CG, Britto RR, Parreira VF. Relationship between cough strength and functional level in elderly. Rev Bras Fisioter 2010;14(6):470-476.

4. Ishikawa Y, Bach JR, Komaroff E, Miura T, Jackson-Parekh R. Cough augmentation in Duchenne muscular dystrophy. Am J Phys Med Rehabil 2008;87(9):726-730.

5. Smith JA, Aliverti A, Quaranta M, McGuinness K, Kelsall A, Earis J, Calverley PM. Chest wall dynamics during voluntary and induced cough in healthy volunteers. J Physiol 2012;590(3):563-574.

6. Brito MF, Moreira GA, Pradella-Hallinan M, Tufik S. Air stacking and chest compression increase peak cough flow in patients with Duchenne muscular dystrophy. J Bras Pneumol 2009;35(10):973979.

7. Bach JR. Mechanical insufflation-exsufflation. Comparison of peak expiratory flows with manually assisted and unassisted coughing techniques. Chest 1993;104(5):1553-1562.

8. Bach JR, Mahajan K, Lipa B, Saporito L, Goncalves M, Komaroff E. Lung insufflation capacity in neuromuscular disease. Am J Phys Med Rehabil 2008;87(9):720-725.

9. Kang SW, Bach JR. Maximum insufflation capacity: vital capacity and cough flows in neuromuscular disease. Am J Phys Med Rehabil 2000;79(3):222-227.

10. Fauroux B, Guillemot N, Aubertin G, Nathan N, Labit A, Clément A, Lofaso F. Physiologic benefits of mechanical insufflation-exsufflation in children with neuromuscular diseases. Chest 2008;133(1): 161-168.

11. Winck JC, Gonçalves MR, Lourenço C, Viana P, Almeida J, Bach JR. Effects of mechanical insufflation-exsufflation on respiratory parameters for patients with chronic airway secretion encumbrance. Chest 2004;126(3):774-780.

12. Vianello A, Corrado A, Arcaro G, Gallan F, Ori C, Minuzzo M, Bevilacqua M. Mechanical insufflation-exsufflation improves out- 


\section{Acute Physiological Effects of Air Stacking}

comes for neuromuscular disease patients with respiratory tract infections. Am J Phys Med Rehabil 2005;84(2):83-88; discussion 8991.

13. Jenkins HM, Stocki A, Kriellaars D, Pasterkamp H. Breath stacking in children with neuromuscular disorders. Pediatr Pulmonol 2014; 49(6):544-553.

14. Cleary S, Misiaszek JE, Kalra S, Wheeler S, Johnston W. The effects of lung volume recruitment on coughing and pulmonary function in patients with ALS. Amyotroph Lateral Scler Frontotemporal Degener 2013;14(2):111-115.

15. Toussaint M, Boitano LJ, Gathot V, Steens M, Soudon P. Limits of effective cough-augmentation techniques in patients with neuromuscular disease. Respir Care 2009;54(3):359-366.

16. Bach JR, Zhitnikov S. The management of neuromuscular ventilatory failure. Semin Pediatr Neurol 1998;5(2):92-105.

17. Bianchi C, Carrara R, Khirani S, Tuccio MC. Independent cough flow augmentation by glossopharyngeal breathing plus table thrust in muscular dystrophy. Am J Phys Med Rehabil 2014;93(1):43-48.

18. Dohna-Schwake C, Ragette R, Teschler H, Voit T, Mellies U. IPPBassisted coughing in neuromuscular disorders. Pediatr Pulmonol 2006; 41(6):551-557.

19. Panitch HB. Airway clearance in children with neuromuscular weakness. Curr Opin Pediatr 2006;18(3):277-281.

20. Aliverti A, Pedotti A. Opto-electronic plethysmography. Monaldi Arch Chest Dis 2003;59(1):12-16.

21. American Thoracic Society/European Respiratory Society. ATS/ERS statement on respiratory muscle testing. Am J Respir Crit Care Med 2002;166(4):518-624.

22. Pereira CA, Sato T, Rodrigues SC. Novos Valores De Referência Pra Espirometria Forçada Em Brasileiros Adultos De Raça Branca. J Bras Pneumol 2007;33(4):397-406.

23. Neder JA, Andreoni S, Lerario MC, Nery LE. References values for lung function tests: maximal respiratory pressures and voluntary ventilation. Braz J Med Biol Res 1999;32(6):719-727.

24. Héritier F, Rahm F, Pasche P, Fitting JW. Sniff nasal inspiratory pressure: a noninvasive assessment of inspiratory muscle strength. Am J Respir Crit Care Med 1994;150(6):1678-1683.

25. Araújo PR, Resqueti VR, Nascimento Junior J, Carvalho Lde A, Cavalcanti AG, Silva VC, et al. Reference values for sniff nasal inspiratory pressure in healthy subjects in Brazil: a multicenter study. J Bras Pneumol 2012;38(6):700-707.

26. Aliverti A, Dellacà R, Pelosi $\mathrm{P}$, Chiumello D, Gatihnoni L, Pedoti A. Compartmental analysis of breathing in the supine and prone positions by optoelectronic plethysmography. Ann Biomed Eng 2001; 29(1):60-70

27. Cala SJ, Kenyon CM, Ferrigno G, Carnevali P, Aliverti A, Pedotti A, et al. Chest wall and lung volume estimation by optical reflectance motion analysis. J Appl Physiol 1996;81(6):2680-2689.

28. Lanini B, Masolini M, Bianchi R, Binazzi B, Romagnoli I, Gigliotti F, Scano G. Chest wall kinematics during voluntary cough in neuromuscular patients. Respir Physiol Neurobiol 2008;161(1):62-68.
29. Lanini B, Bianchi R, Binazzi B, Romagnoli I, Pala F, Gigliotti F, et al. Chest wall kinematics during cough in healthy subjects. Acta Physiol 2007;190(4):351-358.

30. Aliverti A, Iandelli I, Duranti R, Cala SJ, Kayser B, Kelly S, et al. Respiratory muscle dynamics and control during exercise with externally imposed expiratory flow limitation. J Appl Physiol 2002; 92(5):1953-1963.

31. Cohen J. Statistical power analysis for the behavioral sciences. 2nd edition. Hillsdale, New Jersey: Lawrence Erbaum; 1988:274-356.

32. Sivasothy P, Brown L, Smith IE, Shneerson JM. Effect of manually assisted cough and mechanical insufflation on cough flow of normal subjects, patients with chronic obstructive pulmonary disease (COPD), and patients with respiratory muscle weakness. Thorax 2001;56(6):438-444.

33. Jeong JH, Yoo WG. Effects of air stacking on pulmonary function and peak cough flow in patients with cervical spinal cord injury. J Phys Ther Sci 2015;27(6):1951-1952.

34. Lo Mauro A, D’Angelo MG, Romei M, Motta F, Colombo D, Comi GP, et al. Abdominal volume contribution to tidal volume as an early indicator of respiratory impairment in Duchenne muscular dystrophy. Eur Respir J 2010;35(5):1118-1125.

35. Romei M, Mauro AL, D'Angelo MG, Turconi AC, Bresolin N, Pedotti A, Aliverti A. Effects of gender and posture on thoracoabdominal kinematics during quiet breathing in healthy adults. Respir Physiol Neurobiol 2010;172(3):184-191.

36. Lindholm P, Nyren S. Studies on inspiratory and expiratory glossopharyngeal breathing in breath-hold divers employing magnetic resonance imaging and spirometry. Eur J Appl Physiol 2005;94(5):646651.

37. Seccombe LM, Rogers PG, Mai N, Wong CK, Kritharides L, Jenkins CR. Features of glossopharyngeal breathing in breath-hold divers. J Appl Physiol 2006;101(3):799-801.

38. Loring SH, O'Donnell CR, Butler JP, Lindholm P, Jacobson F, Ferrigno M. Transpulmonary pressures and lung mechanics with glossopharyngeal insufflation and exsufflation beyond normal lung volumes in competitive breath-hold divers. J Appl Physiol 2007; 102(3):841-846.

39. Aliverti A, Cala SJ, Duranti R, Ferrigno G, Kenyon CM, Pedotti A, et al. Human respiratory muscle actions and control during exercise. J Appl Physiol 1997;83(4):1256-1269.

40. Illi SK, Hostettler S, Aliverti A, Spengler CM. Compartmental chest wall volume changes during volitional hyperpnoea with constant tidal volume in healthy individuals. Respir Physiol Neurobiol 2013; 185(2):410-415.

41. Aliverti A, Kayser B, Macklem PT. A human model of the pathophysiology of chronic obstructive pulmonary disease. Respirology 2007;12(4):478-485.

42. Sarmento A, Resqueti VR, Fregonezi GA, Aliverti A. Assessment of gas compression and lung volume during air stacking maneuver. Eur J Appl Physiol 2016 [Epub ahead of print]. doi: 10.1007/s00421016-3511-y. 